\title{
La etiqueta ambiental: regulación en el derecho Cubano
}

\author{
The environmental label: regulation in the Cuban right
}

\author{
Alcides F. Antúnez Sánchez a, ${ }^{\text {, }}$ Eduardo Díaz Ocampo ${ }^{\text {b }}$ \\ a Universidad de Granma, Cuba

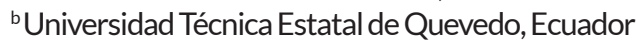

\section{RESUMEN}

El artículo muestra las insuficiencias dentro de la ordenación jurídica cubana de la concesión de la etiqueta ambiental por la Administración Pública, entregadas de forma mayoritaria a las formas de gestión del sector estatal en el tráfico mercantil. Fueron empleados para ello los métodos de investigación teórico-jurídico, el histórico-lógico, el exegético-jurídico, el de derecho comparado, y el de análisis y síntesis. Se tiene como conclusiones que el empresario cubano no tiene aún una cultura ambiental adecuada, que pondere el uso de la etiqueta-certificación ambiental dentro de las formas de gestión, que les permita el acceso a mercados más exigentes dentro y fuera de la nación; incidiendo de forma desfavorable la obsolescencia y heterogeneidad de la empresa y la industria con más de 50 años de explotación; elemento válido para el consumidor, el que tampoco goza de una cultura adecuada en materia de certificación ambiental. La Contraloría de la República de Cuba, al amparo de lo que se dispone en el Acuerdo del Consejo de Estado de 2010, como Reglamento de la Ley $N^{\circ} 107$ de 2009 de este órgano supremo de control no establece la certificación ambiental al concluir la auditoría ambiental. La Administración Pública cubana continúa ponderando la calidad y no la certificación ambiental, lo que impide conformar la empresa amigable con el ambiente dentro de las formas de gestión en el siglo XXI. En contradicción con lo que se reconoce en la doctrina y en la legislación foránea respecto a la etiquetacertificación ambiental.

Palabras claves: Inspección, auditoría, consumidores ecológicos, empresa responsable.

\section{ABSTRACT}

The article shows the gaps within the Cuban legal arrangement in the concession of the environmental labels by Public Administration, gives in majority way to the forms of public management in the mercantile traffic sector. It was used for the method, types of research as: theoretical and legal, historical and logical, legal exegetical, comparative law, and analysis and synthesis. Concluding that Cuban's businessman does not have yet a proper environmental culture, which examine the use of the certification of environmental label within ways of management, which allows them access to markets with more demand, inside and abroad of the nation; affecting in unfavorable way to obsolescence and heterogeneity of the company also to industry with over 50 years of exploitation; valid element for consumers, who also don't have an appropriate environmental certification culture. The Comptroller of the Republic of Cuba, on the basis of the Council of State agreement from 2010, as in law $N^{\circ} 107$ from 2009 establish from the same supreme body of control; does not establish an environmental certification from environmental audit. Cuban public administration continues pondering quality and not environmental certification, which enable to conform a company environmental friendly within them management ways in the XXI century. In contradiction with what is recognized in the doctrine and the foreign legislation regard to environmental label certification.

Keywords: Inspection, audit, ecologic consumers and responsible company.

Historial del artículo:

Recibido, 20 de octubre de 2015; aceptado, 12 de febrero de 2016; disponible en línea, 30 de marzo de 2016

* Máster en Asesoría Jurídica profesor auxiliar de la Facultad de Ciencias Económicas y Sociales de la Universidad de Granma, República de Cuba.

Correo: aantunez@udg.co.cu

Este es un artículo de acceso abierto, licencia CC BY (https://creativecommons.org/licenses/by/4.0/) 


\section{INTRODUCCIÓN}

La preocupación por la protección del medio ambiente por parte de los gobiernos, de los empresarios en las formas de gestión y de los ciudadanos como consumidores de los bienes y servicios ha ido en aumento desde la primera década del siglo XXI como consecuencia del cambio climático y de las adversidades que este trae consigo lo que ha hecho necesario el desarrollo de herramientas que permitan a la administración pública ejecutar el control ambiental a través de la auditoría pública y la potestad inspectora.

En este sentido, se aprecia como en los últimos años diversos estudios recogen la creciente disposición de los consumidores a pagar por productos que alcanzan la categoría verde. Es decir, productos que tienen un menor impacto ambiental que los productos convencionales similares, ya sea, por la utilización en su proceso de producción de tecnologías que generan menos emisiones contaminantes o residuos (tecnologías limpias).

Por ello, los autores valoran que, desde el punto de vista de las empresas, esta predisposición de los consumidores genera incentivos a la inversión en tecnologías limpias siempre que ello se traduzca en una mayor demanda de sus productos.

Es así que se creó la Red de Etiquetado Ecológico Mundial (GEN) como asociación no lucrativa de organizaciones de etiquetado ambiental de tercera parte en 1994 para mejorar, promover y desarrollar el ecoetiquetado de los productos y los servicios desde el pasado siglo.

En la actualidad es la Asociación Española de Normalización y Certificación (AENOR) quien preside la red mundial del etiquetado ecológico para certificar las formas de gestión. Se aprecia cómo han sido creados mecanismos de respuestas a través de instrumentos que permiten la tutela ambiental a la Administración Pública con la implementación de las herramientas de gestión, en particular con la ejecución de la auditoría ambiental. Su finalidad es velar por la protección del medio ambiente como bien público.

Al concluir la evaluación a la forma de gestión auditada se le entrega la etiqueta-certificación ambiental, reconocida como el instrumento voluntario dirigido a la protección ambiental, factible económicamente y donde participa un equipo multidisciplinario para determinar su concesión por su multidimensionalidad.

Es concebido justiprecian los autores como un instrumento administrativo cuya naturaleza es reglada dentro de los ordenamientos jurídicos. Representa en sí un estándar de competencia económica para el desarrollo sostenible a las formas de gestión en el comercio como uno de los valores agregados que aporta.

El objetivo que se persigue con la implementación de un sistema de etiqueta ambiental en un país es proteger a los consumidores, es denominada en la doctrina jurídica como certificación ambiental o ecoetiquetado de forma indistinta; como sistema persigue sensibilizar a los consumidores para la adquisición de productos cuya repercusión ambiental sea mínima. Es un estímulo para que las formas de gestión dirijan su producción y distribución en la red de comercio de bienes y servicios con la implementación de tecnologías limpias, para obtener ventajas competitivas.

Su génesis se constata por los autores que ocurrió en Alemania a partir de 1977 del pasado siglo como su antecedente mundial, se identificó con el Ángel Azul, por lo que le corresponde el mérito de ser la primera nación que la implementó por la Administración Pública en sus formas de gestión. El ejemplo alemán fue seguido en 1989 por Canadá y Japón.

Este sistema de etiquetado continúo su implementación en otros países como Noruega, Suecia, Finlandia, Austria, Francia, Portugal y Nueva Zelanda; se han insertado en la consumación de este ecoetiquetado y han elaborado sus propios sistemas para aplicarlo a partir de 1989.

Por ello, se aprecia como la expansión del ecoetiquetado ha tomado una relevancia creciente, toda vez que la Comisión de Comercio y Medio Ambiente de la Organización Mundial del Comercio ha reconocido que los programas de ecoetiquetado pueden ser valiosos instrumentos de política ambiental para cada nación.

Disímiles han sido los modos de manifestación y protección del ecoetiquetado con mayor o menor intervención pública, pero convergen en la finalidad de estimular el principio del desarrollo sostenible en la actividad económica con un rol protagónico y simbiosis en la relación Estado-Empresa.

Sin embargo, consideran los autores que aún queda camino por andar en la implementación general y en la eficacia de los propósitos que se persigue con el etiquetado ambiental, donde la contribución de los ordenamientos jurídicos es trascendental para su regulación; mientras unos van a la vanguardia otros están rezagados, lo cual obstaculiza la internacionalización racional del sistema de certificación ambiental y el conocimiento adecuado por los consumidores. 


\section{La etiqueta-certificación ambiental en cuba}

El siglo XXI inicia dando respuestas a los daños ambientales originados por el cambio climático, trae aparejado que en las diversas áreas geográficas del planeta se preparen los Estados para responder de manera adecuada a este fenómeno natural a través de políticas ambientales "paradigmas".

Cuba como país insular no está ajeno a tal responsabilidad, para ello desde el pasado siglo XX la calidad de los bienes y los servicios es certificada por el Ministerio de Ciencia, Tecnología y Medio Ambiente (CITMA), al amparo de lo que se dispone en la Ley $N^{\circ} 81$ de 1997. Hasta ahora se ha dirigido al sector estatal como forma de gestión predominante del país, razón por la cual será el principal enfoque de este análisis.

Esta etiqueta-certificación ambiental ha estado destinada esencialmente al sector turístico, como respuesta estratégica, toda vez que a mediados del siglo XX el turismo se convirtió en un sector significativo para la sociedad mundial y la nación cubana consideró invertir de conjunto con el capital extranjero en la denominada industria sin humo.

La nación al concluir la década de los 90 del pasado siglo fomentó el desarrollo turístico, como sector multifacético y de interés económico para el país, se le denominó en ese momento la locomotora de la economía cubana, la que ha seguido continuando su fortalecimiento en este siglo XXI, a pesar de las adversidades del bloqueo económico.

En sí, la actividad económica del turismo comprende una serie de prestaciones cuyo rasgo común más destacado es su predisposición a satisfacer las necesidades peculiares del sujeto que se halla temporalmente fuera del lugar donde reside de forma habitual, por lo que se corresponde con el ecoetiquetado de servicios al momento de su adquisición por los consumidores, en atención a que el turismo foráneo si tiene una cultura en el consumo de este tipo de productos.

En Cuba se valora que se distinguen dos programas principales: el Aval ambiental y el Reconocimiento Ambiental Nacional, ambos del Ministerio de Ciencia, Tecnología y Medio Ambiente (CITMA) quien es el organismo de la Administración Pública que hace su entrega pública a las formas de gestión. En este proceso de inspección ambiental, se les evalúa el desempeño ambiental en las empresas y constituye un imperativo cuando se trabaja por una certificación; la norma cubana NC-ISO 14031 se refiere a este proceso evaluativo como: "... un proceso para facilitar las decisiones de gestión con respecto al desempeño ambiental por medio de indicadores, recopilando y analizando datos, evaluando información, reportando, comunicando, revisando periódicamente y mejorando este proceso"...

Esta norma señala que la Evaluación de Desempeño Ambiental (EDA) integra tres dimensiones de análisis con indicadores propios: el comportamiento operacional que refleja los resultados ambientales de una entidad, los indicadores de gestión que recogen los esfuerzos que realiza la organización en materia ambiental y los indicadores de situación ambiental que caracterizan el entorno en que se encuentra enclavada la organización.

Los autores valoran que la socialización de la información ambiental no es solo una demanda o una aspiración de los científicos, los técnicos y de los especialistas dedicados a este temática, constituye en sí una necesidad y una contribución a los procesos de mejora que desarrollan las organizaciones empresariales en las formas de gestión para conocer su posicionamiento respecto a la competencia y a las tendencias más reconocidas en el escenario en el cual se desenvuelven, no concebido de manera adecuada por el sector estatal cubano, al ser este el de mayor predominio en el escenario mercantil nacional. Que quizás sea estiman los autores porque solo la producción científica se ha dirigido desde la arista económico-contable y exista poca producción científica desde la ciencia del Derecho y por qué no desde otras ciencias por su multidimensionalidad.

La Constitución Cubana de 1976, en relación con estos imperativos, omite la referencia directa a las actividades que por su especial naturaleza se consideran como servicios públicos en la nación, cuya titularidad estatal le imprime un régimen jurídico especial. Se entraña una norma que delimite de forma taxativa cuáles son los servicios públicos que debe garantizar el Estado, sea prestándolos de forma directa o mediante terceros, sobre la base del uso de construcciones jurídico-administrativas (concesiones y contratos). No obstante, aun cuando no existe reseña expresa de ellos, algunos se pueden colegir de la lectura del texto constitucional, a saber: educación, salud, transporte, comunicaciones, entre otros.

Los autores consideran añadir lo preceptuado en los artículos constitucionales 10 y 27 , sobre la protección del medio ambiente para lograr la sostenibilidad ambiental y la necesidad de cumplir con la legislación en la materia ambiental, la necesidad de actualizar la normativa ambiental en la nación cubana.

Empero, la situación en Cuba a partir de lo establecido en el Acuerdo 5179 del Comité Ejecutivo del Consejo de Ministros del 2004 cuando se decidió organizar, autorizar, ejecutar y controlar, según procedió los trabajos de certificación de conformidad de los 
productos, los sistemas de gestión de la calidad, los sistemas de gestión ambiental, los sistemas de gestión de seguridad y salud del trabajo, software, el etiquetado ambiental, la seguridad eléctrica, personas, así como de otros procesos y servicios de importancia para el comercio y la calidad de vida de la población.

Ello permitió establecer, otorgar y retirar las marcas y certificados de conformidad por ella emitidos. Aprobar o denegar la solicitud de otras organizaciones como órganos de certificación de tercera parte, según las atribuciones establecidas en el Decreto Ley $\mathrm{N}^{\circ} 182$ de 1998, para conformar valoran los autores a futuro el Reglamento General de la etiqueta ambiental de la República de Cuba, con su adecuado reconocimiento dentro de la Ley de Empresas y en la Ley de protección y derechos del consumidor, ausentes en el ordenamiento jurídico patrio.

En Cuba, aprecian los autores la existencia de dos esquemas fundamentales que certifican el trabajo ambiental en la industria hotelera, uno el Aval Ambiental y el Reconocimiento Ambiental Nacional (RAN). El RAN es más exigente en cuanto a indicadores y criterios de evaluación. Se rigen por la Resolución $\mathrm{N}^{\circ}$ 27 de 2000 del CITMA y esta estableció dos categorías para su otorgamiento: el Reconocimiento Ambiental Nacional a Nivel Básico y el Sello Distintivo.

Terry Berro (1), consideró que el CITMA, mediante la Resolución № 27 de 2000, se puso en vigor el Sistema Nacional de Reconocimiento Ambiental con los objetivos siguientes: distinguir a las entidades ocupadas en la solución de sus problemas ambientales, incentivar la mejora continua del desempeño económico y ambiental de las mismas y prepararlas para la obtención de certificaciones internacionales, cuando existieran condiciones para ello. Cuatro años después, la Resolución № 135 de 2004 del CITMA derogó a la anterior y comenzó a regir el proceso, aun cuando persigue objetivos similares, garantiza un mejor ordenamiento de los procedimientos y propicia la inserción de conceptos en el ámbito internacional como el de Producción Más Limpia.

Este acto administrativo del Ministerio de Ciencia, Tecnología y Medio Ambiente (CITMA), permite prioridades en la aplicación del instrumento, establece penalizaciones, dispone mecanismos reclamatorios, y establece políticas de seguimiento y control. Reconoce que aun y cuando este sistema esta implementado existen dificultades que atentan contra los propósitos de lograr una mayor incorporación del sector estatal al proceso. Entre ellas el incumplimiento de las regulaciones ambientales vigentes; la baja disponibilidad de recursos materiales y financieros para la realización de los diagnósticos ambientales y la ejecución de alternativas de solución a los problemas identificados; la calidad insatisfactoria de muchos de los diagnósticos presentados; así como el aún insuficiente nivel de concientización de los directivos de muchas entidades en lo relativo a la estrecha vinculación entre desempeño ambiental y desempeño económico. Siendo los punteros en esta certificación el sector del Turismo, algunas empresas estratégicas y el de prestación de servicios públicos.

Se constata, que por parte de la Organización Mundial de la Propiedad Industrial (OMPI), se legitimó la etiqueta ambiental cubana en el 2008, a solicitud del CITMA; se daba así la Isla otro paso vital en el propósito de contar con un atributo que dé a sus productos un lugar de preferencia en el cada vez más competitivo comercio internacional. Pero este no es el único beneficio que tendrán la economía, sus productos y servicios, y los consumidores, tanto extranjeros como nacionales.

Martín-Mateo R. (2), consideró que la etiqueta ambiental es una marca de conformidad que certifica que los productos están acordes con las Normas Cubanas $y$, en su ausencia, con los documentos referentes a la protección del medio ambiente. Permitirá otorgar un distintivo para la diferenciación de los productos «verdes» (ecológicos) y el enfrentamiento de posibles barreras al comercio internacional, así como proporcionar a los consumidores mejor información sobre las repercusiones ambientales de los artículos, sin comprometer por ello la seguridad de estos o de los trabajadores, ni afectar las propiedades que hacen que una mercancía esté apta para el consumo.

Significa que el país está en condiciones para desarrollar e implementar el Programa de Etiquetado Ambiental de la República de Cuba, mecanismo a través del cual se pretende promover el diseño, elaboración, comercialización y utilización de productos con efectos negativos mínimos sobre el medio ambiente en todo su ciclo de vida. Es el escenario favorable para desarrollar e implementar tan importante y necesario instrumento, a partir de la base reglamentaria y normativa, la reanimación de la economía y la atención priorizada que ofrece el gobierno a la temática ambiental.

En su condición de experto consideró que la etiqueta se basa en el Análisis del Ciclo de Vida del producto (ACV) y supone la concesión de uso de una marca en caso de que el producto o familia de estos cumpla con los criterios ecológicos específicos. Se tendrán en cuenta los elementos que puedan originarse en el ciclo de vida del producto o familia de productos en cuestión, los cuales considerarán la contaminación y degradación del suelo, el agua, la atmósfera, el ruido, el consumo de energía y recursos naturales como el hídrico, así como la repercusión en los ecosistemas. Las pautas ecológicas estarán a disposición pública con toda transparencia y tendrán validez por tres años. 
Las novedades relacionadas con la preservación ecológica han estado, en los últimos años, en las entidades turísticas del país, en particular las instituciones hoteleras, que se interesan por contar en sus servicios de alojamiento con la distinción comercial al estilo de la que puede ofrecerles una etiqueta ambiental. Con la aplicación de este programa deberá asegurar a la economía nacional un vehículo que propicie la mejora continua del desempeño ambiental en todo el ciclo de vida de los productos que obtengan la etiqueta, la cual puede ser solicitada por cualquier persona natural o jurídica que justifique su condición de representante legal de la empresa que fabrica el producto o presta el servicio. Se excluye de solicitar esta etiqueta los medicamentos y los alimentos, los cuales disponen de otros sistemas de certificación que avalan sus cualidades, según normas internacionales.

La introducción de estos resultados es de gran importancia ya que muchas empresas preocupadas por el impacto que generan sus actividades sobre el medio natural y el cumplimiento de la legislación ambiental vigente, comienzan por certificar su SGA, y luego se interesan y preocupan por etiquetar sus productos para lo cual podrán ahora acogerse al Programa de Etiquetado Ambiental.

De aquí que se siga ponderando la necesidad de conformar empresas responsables con el ambiente por parte de los autores en el escenario mercantil patrio. Los beneficios serán relevantes tanto en lo económico, lo político como en lo social, ya que en un país donde predomina la voluntad de protección del medio natural, ello contribuye a elevar el nivel de conciencia ambiental y la preferencia por los productos que obtengan la Etiqueta. El programa también contribuye a incrementar la competitividad de las exportaciones con empresas amigable con el ambiente. Con la oficialización internacional de la etiqueta, Cuba se convierte en el tercer país en la región que cuenta con un programa de este tipo.

La etiqueta ambiental de un producto a partir del programa nacional debe responder a los criterios establecidos por el país, ser transparente y sometida a acuerdos bilaterales y/o multilaterales de reconocimiento, como puede ser el de la Red Mundial de Eco-etiquetado, conocida por sus siglas en inglés: GEN. Los Programas de Etiquetado Ambiental Tipo I no son muy abundantes en América Latina. Existen en Estados Unidos, Europa y Asia, y forman parte de la Red Mundial de Eco-etiquetado, con 32 países miembros y sede rotativa, la cual radica ahora en Japón.

Se analiza por los autores, que, a más de un decenio del siglo XXI, de aparecer entre los órganos del Estado la Contraloría General de la República como el órgano superior de control, regularse la auditoría ambiental en el Reglamento de la Ley $N^{\circ} 107$ de 2010, no se estableció la certificación ambiental de los bienes y prestación de servicios dentro de las formas de gestión luego de ejecutarse la función auditora. A pesar que el CITMA lo ha venido ejecutando a través de la potestad inspectora en la nación, solo que en un pequeño sector de interés económico como se ha citado en el desarrollo del artículo.

Se valora en el plano jurídico que la auditoría ambiental patria se omitió por parte del legislador la certificación ambiental al concluir el proceso evaluador, usada en el resto de la doctrina consultada en la región de Iberoamérica y en el resto de países constatados por los beneficios que como valor agregado trae para el comercio interno y el foráneo a los empresarios de las formas de gestión estatal y privada para transitar de la certificación de la calidad hacia la ambiental.

También se aprecia como en la nación cubana dentro del derecho interno el control ambiental se ejecuta a través de la potestad inspectora, la fiscalización, el control gubernamental y la auditoría ambiental, en relación con los principios rectores del Derecho Ambiental por los órganos y organismos del Estado a través de la obtención de los títulos habilitantes o por designación como funcionarios públicos en los organismos de la Administración Pública que controlan la política ambiental patria.

Con la actualización del modelo económico cubano en el siglo XXI, se considera que demanda un éxito empresarial que permita la sustitución de importaciones, lo que significa crear riquezas, las que no podrán ser alcanzadas sin que todas las esferas marchen de forma coordinada y no avancen unas en detrimento de otras.

La década del 80 del siglo pasado, señaló que el éxito de la empresa estatal se alcanzaba con el logro de la marca estatal de calidad de sus productos, el actual siglo mide no solo en volúmenes productivos sino la eficiencia y eficacia de la gestión lo que implica que ningún subsistema sea afectado, la que no podrá ser evaluada sí no se aplica la auditoría ambiental por la CGR a las formas de gestión del sector estatal por ser estas las mayoritarias y se certifiquen ambientalmente.

Sanz Larruga (3), señalaba "la empresa estaba acostumbrada al conflicto intraempresario -es decir, al conflicto entre patrón, empleador y trabajador, conflicto de derecho del trabajo- y al conflicto inter-empresario -de derecho comercial, conflicto con el cliente y con el proveedor-. De pronto se ve inmersa en otro tipo de conflicto, de base social, porque el Derecho Ambiental es un derecho de grupo, de clase, de categoría, ligado a una pluralidad de sujetos integrantes de un grupo, vinculados en virtud de una pretensión de goce de una misma prerrogativa, al ser un derecho de goce, 
disfrute y afectación solidaria".

Antúnez Sánchez (4), señaló que la política del Estado y del Gobierno en el siglo XXI entroniza la esfera ambiental no solo como escenario de todas las actividades del país, sino como el preciado objeto de protección y conservación para lograr la sostenibilidad en la actualización del modelo económico. Se destaca por este autor que el logro empresarial en estos momentos de actualización del modelo económico se alcanzará con el reconocimiento dentro de las formas de gestión estatal en el sector estatal cubano de su condición ambiental y no solo por el respeto y el cumplimiento de los planes técnico-económicos como se ponderaron en el pasado siglo XX.

Desde estos enfoques, justiprecian los autores que el éxito empresarial de las formas de gestión del sector estatal no se deberá tener en cuenta solo por cifras de los productos finales y la satisfacción de las necesidades del país, sino en la calidad del proceso productivo en el que la dimensión ambiental esté presente y deba ser altamente considerada al momento de entregar la certificación ambiental al concluirse la auditoría.

En Cuba, los Lineamientos de la Política Económica y Social del Partido y la Revolución, cuyo fin es actualizar el modelo económico como objetivo para garantizar la continuidad e irreversibilidad del sistema social, contribuirá al desarrollo económico y a la vez elevar el nivel y calidad de vida de la población conjugados con la necesaria formación de valores éticos y políticos de los ciudadanos, por ser considerados de suma importancia la práctica de la auditoría ambiental como meta para lograr el desarrollo sostenible y el bienestar social.

Todas estas formulaciones, permiten tomar posición a los autores en relación al desarrollo de la forma de gestión estatal cubana con la empresa amigable con el ambiente integradora de la política dentro de la actualización del modelo económico en el sector estatal cubano, donde la implementación de las herramientas de gestión en su conjunto por parte de la Contraloría General de la República (CGR) y el CITMA juegan un rol esencial para que el país promueva la certificación ambiental con la etiqueta ambiental, pero a criterio de los autores le corresponderá a la CGR esta decisión, toda vez que el CITMA es quien acompañará en esta certificación a la CGR, tal y como se dispone en la Ley N 107 de 2009 y su Reglamento del 2010.

Para ello se estima que la ejecución de la auditoría ambiental permitirá lograr empresas eficientes y competitivas en el mercado al aplicarse los resultados de la ciencia, las tecnologías, la innovación en el cuidado de los recursos naturales a través de acciones protectoras del entorno, del patrimonio y de la cultura de la nación cubana.
Para ello es necesario continuar el trabajo desarrollado en base a perfeccionar las condiciones organizativas, las jurídicas e institucionales que se emplean para establecer los tipos de organizaciones económicas que garanticen la combinación de la fórmula I+D $+C+(E A)$ para lograr un desarrollo rápido y eficaz de nuevos productos y servicios, con estándares de calidad y de la gestión comercializadora interna y exportadora, requeridos hoy de una evaluación por esta tipología de auditoría que permita concluir con la certificación ambiental, factores estos que contribuirán a una mejor apertura dentro del mercado en materia de competencia a las formas de gestión del sector estatal cubano como valor agregado a través de empresas amigables con el ambiente.

En Cuba, Fernández Infante (5) desde la Oficina de Normalización del CITMA ha señalado la necesidad e importancia de que las formas de gestión se inserten en el sistema de etiquetado-certificación ambiental, por su valor para el comercio y la protección del medio ambiente. Otros autores desde las ciencias contables como Fernández Companioni (6) y Triana Cordoví (7), señalaron la necesidad de que las formas de gestión en la nación cubana transiten a otro nivel, el de la calidad ambiental hacia la certificación ambiental.

Antúnez Sánchez (4), consideró que le corresponderá definir la política tecnológica para reorientar el desarrollo industrial de la nación, el control de las tecnologías en uso en el país y las nuevas que sean adquiridas, a fin de promover su modernización sistemática con atención a los análisis de la eficiencia energética, la eficacia productiva y el impacto ambiental que estas puedan generar, factores estos que contribuirán a elevar la soberanía tecnológica en las ramas estratégicas en la actualización del modelo económico del sector estatal.

No obstante, hay organismos de la Administración Pública en la nación cubana que dan pasos de avances en este sentido como son el Ministerio de Turismo, la Industria productora de medicamentos, la industria eléctrica y la del petróleo.

Pero aún valoran los autores que no ha sido asimilado de manera adecuada la implementación de la etiqueta certificación ambiental por el Ministerio de la Agricultura (implementación de las agroecologías), la Industria de alimentos, entre otros. Lo que permitirá seguir profundizando a futuro sobre la relevancia de este tema que permita con ello el progreso económico a Cuba en su actualización de su modelo económico.

\section{CONCLUSIONES}

El sistema de referencia mundial ha sido la etiqueta del Ángel Azul, cuyo origen fue en Alemania, data de 
más de treinta años de existencia en su aplicación. Fue creada con el objetivo de proteger al bien público ambiental, de aquí que muchos Estados lo tomen como referente dentro de sus sistemas aplicados a las formas de gestión estatales o privadas. Ello permite establecer el crecimiento económico en correspondencia con el nivel de calidad de vida del ser humano y la preservación del medio ambiente.

El interés creciente de los consumidores para adquirir productos respetuosos con el ambiente, permite la oportunidad de su presencia en los mercados para que las formas de gestión ejecución su certificación como empresa amigable con el ambiente para ser más competitivas, ejecutada a través de la auditoría ambiental. Siendo el objetivo primordial obtener beneficios económicos, pero con regulación y control, que permita reforzar la imagen de la marca dentro del marketing ecológico.

Las formas de gestión en el mercado tienen en cuenta que las Administraciones Públicas participan en la entrega de la concesión de la etiqueta ecológica, otorgando su reconocimiento oficial a través del distintivo aprobado por cada país. Lo que le permite posicionarse de forma más ventajosa en la contratación pública en la lucha por proteger al medio ambiente.

La auditoría ambiental emergió debido a la contaminación empresarial y su enfrentamiento por el Estado, su génesis fue la auditoría social. Se consolida posteriormente como acción de control practicada por las Entidades Fiscalizadores Superiores y las Sociedades Mercantiles, posee una construcción jurídica a partir de estar reconocida en Convenios y Acuerdos Internacionales, es introducida en los derechos internos mediante los textos constitucionales, las leyes ambientales y de contralorías: brinda solidez y sustento para el desarrollo de políticas ambientales como la herramienta de gestión ambiental. Puede ser voluntaria u obligatoria y manifestarse como una actividad policía, un servicio público o actividad de fomento acorde a su ejecución y consecuencias. Vinculada al etiquetado-certificación ambiental.

La etiqueta-certificación ambiental en el ordenamiento jurídico cubano es un acto administrativo cuya potestad recae en el Ministerio de Ciencia, Tecnología y Medio Ambiente, organismo de la Administración Pública que al amparo de la potestad inspectora hace la entrega a las formas de gestión estatal del país consideradas como sujetos estratégicos para el desarrollo del comercio internacional en la nación a través de este reconocimiento. Su naturaleza jurídica es reglada. Se sustenta en la Ley $N^{\circ} 81$ de 1997 y en la Resolución N ${ }^{\circ}$ 135 de 2004, como en las normas complementarias ISO (tipo I).

El empresario cubano no tiene aún una cultura ambiental adecuada, que pondere el uso de la etiqueta-certificación ambiental dentro de las formas de gestión, que les permita el acceso a mercados más exigentes dentro y fuera de la nación. Incidiendo de forma desfavorable la obsolescencia y heterogeneidad de la empresa y la industria con más de 50 años de explotación. Elemento válido para el consumidor, el que tampoco goza de una cultura adecuada en materia de certificación ambiental.

La Contraloría de la República de Cuba, al amparo de lo que se dispone en el Acuerdo del Consejo de Estado de 2010, como Reglamento de la Ley $N^{\circ} 107$ de 2009 de este órgano supremo de control no establece la certificación ambiental al concluir la auditoría ambiental. La Administración Pública cubana continúa ponderando la calidad y no la certificación ambiental, lo que impide conformar la empresa amigable con el ambiente dentro de las formas de gestión en el siglo XXI. En contradicción con lo que se reconoce en la doctrina y en la legislación foránea respecto a la etiqueta-certificación ambiental.

\section{REFERENCIAS BIBLIOGRÁFICAS}

1. Terry C. Reconocimiento Ambiental Nacional. Cinco años de experiencia. Revista electrónica de la Agencia del Medio Ambiente. 2005; (9): 1-7.

2. Martín-Mateo R. El etiquetado ecológico, nuevo instrumento para la tutela ambiental. España: Editorial Trivium; 1994.

3. Sanz I. Sobre la naturaleza jurídica de la etiqueta ecológica. España: Editorial Marcial Pons; 2000.

4. Antúnez AF. La auditoría ambiental, nexo con la empresa amigable con el ambiente. Desarrollo sostenible. Revista de Auditoría Pública. 2015; (65): 1-20.

5. Fernández S. El sistema comunitario de etiquetado ecológico. Estudios sobre consumo. 1993; (27): 11-27.

6. Fernández-Companioni A. La empresa cubana, la competitividad, el perfeccionamiento empresarial y la calidad. Anuario de la Facultad de ciencias económicas y empresariales. 2012; 4: 3-15

7. Triana J. Cubana: un balance de la transformación. Econ. y Desarrollo. 2014; 151(1): 12-29. 